\title{
Study Activity Cause or Rational
}

National Cancer Institute

\section{Source}

National Cancer Institute. Study Activity Cause or Rational. NCI Thesaurus. Code C70901.

A reason, motivation, or rationale for the activity-instance, such as the patient condition demands an intervention, or a patient requests that a procedure be performed. 